\title{
Analysis of Conditional PDF-Based Split VQ
}

\author{
Saikat Chatterjee, Student Member, IEEE, and T. V. Sreenivas, Senior Member, IEEE
}

\begin{abstract}
The split vector quantization (SVQ) method results in a "coding loss" due to the independent quantization of split subvectors. To recover the coding loss, conditional pdf-based split vector quantization methods were proposed recently.

For a multivariate Gaussian source, we theoretically derive the expression of rate-distortion (R/D) performance for conditional pdf-based split vector quantization (CSVQ) method, using high rate quantization theory and optimum bit allocation. We also derive the rate-distortion performance expression of traditional SVQ method and, thus, quantify the coding gain of CSVQ method over SVQ method.
\end{abstract}

Index Terms-Conditional pdf, high rate quantization, multivariate Gaussian, split vector quantization.

\section{INTRODUCTION}

$\mathbf{V}$ ECTOR quantization (VQ) is a fundamental and widely used source coding method. However, because of its exponential complexity, structured/product code VQ methods are popular [5], which reduce the complexity at the cost of R/D performance. One of the most cited product code VQ methods is split VQ (SVQ) [6] or partitioned VQ [5] which has found wide-scale applications in many speech coders [6]-[8].

In SVQ, the full vector is split into subvectors and then quantized independently. This approach sacrifices the correlation between the subvectors and thus leads to a coding loss, referred to as "split loss" [11]. Using the information-theoretic measures, such as conditional entropy and Kullback-Leibler distance, it is shown that the use of conditional pdf can recover the split loss [11]. We have recently developed a sequential SVQ (SeSVQ) method in [12]; the SeSVQ is a nonparametric method of exploiting the conditional pdf. We have also developed a parametric method of conditional pdf-based SVQ (CSVQ) technique in [13].

In this letter, we theoretically quantify the coding gain of CSVQ over SVQ, in general for a multivariate Gaussian source. The associated formulas are derived using high rate quantization theory and optimum bit allocation. We use square Euclidean distance (SED) as a distortion measure.

\section{PRELIMINARIES}

For a source pdf of $f_{\mathbf{g}}(\mathrm{g})$, the high rate quantization distortion (mean-square error) using a VQ is given by [5]

Manuscript received March 1, 2007; revised May 17, 2007. The associate editor coordinating the review of this manuscript and approving it for publication was Prof. Yimin Zhang.

The authors are with the Department of Electrical Communication Engineering, Indian Institute of Science, Bangalore 560 012, India (e-mail: saikat@ece.iisc.ernet.in; tvsree@ece.iisc.ernet.in).

Digital Object Identifier 10.1109/LSP.2007.901685

$$
\mathcal{D} \geq N^{-\frac{2}{h}} \frac{1}{\pi} \frac{h}{h+2}\left(\frac{h}{2} \Gamma\left(\frac{h}{2}\right)\right)^{\frac{2}{h}}\left[\int_{\mathcal{R}^{h}}\left[f_{\mathbf{g}}(\mathbf{g})\right]^{\frac{h}{h+2}} d \mathbf{g}\right]^{\frac{h+2}{h}}
$$

where $N=2^{b_{g}}$ is the number of Voronoi regions and $b_{g}$ is the allocated bits/vector to quantize the source; $h$ is the dimension of vector $\mathbf{g}$; and $\Gamma(\cdot)$ is the usual gamma function. The (1) provides a formal lower bound that is valid in the domain of fine quantization [4]. The equality holds if and only if the Voronoi regions are approximately spheres in Euclidean norm [2]. As the spheres cannot tessellate $\mathcal{R}^{h}$, the equality does not hold true in general, although it may be approximately true in some cases [2]. In practice, the experimental results close to the lower bound are achievable for standard PDFs at high rate [9], and the equality is used to optimize the R/D performance of a quantizer [9], [10]. Thus, we use the lower bound [equality in (1)] in this letter.

Let us consider the lower bound for a multivariate Gaussian source. Suppose that $f_{\mathbf{g}}(\mathrm{g})$ is multivariate Gaussian distributed as $f_{\mathbf{g}}(\mathbf{g})=\mathbf{N}\left(\mu_{\mathbf{g}}, C_{\mathbf{g}}\right)$. Substituting in (1), we get

$$
\mathcal{D}_{\mathbf{g}}=\left(2^{b_{g}}\right)^{-\frac{2}{h}} K_{h}\left|C_{\mathbf{g}}\right|^{\frac{1}{h}}
$$

where $K_{h}$ is a constant that is dependent only on the value of dimension $h$ as $K_{h}=2((h / 2) \Gamma(h / 2))^{2 / h}((h+2) / h)^{h / 2}$.

Proof: The multivariate Gaussian pdf

$$
\begin{aligned}
& f_{\mathbf{g}}(\mathbf{g})=\mathbf{N}\left(\mu_{\mathbf{g}}, C_{\mathbf{g}}\right)=\frac{\exp \left\{-\frac{1}{2}\left(\mathbf{g}-\mu_{\mathbf{g}}\right)^{T} C_{\mathbf{g}}^{-1}\left(\mathbf{g}-\mu_{\mathbf{g}}\right)\right\}}{(\sqrt{2 \pi})^{h}\left|C_{\mathbf{g}}\right|^{1 / 2}} \\
& \text { Let } K_{I}=\left[\int_{\mathcal{R}^{h}}\left[f_{\mathbf{g}}(\mathbf{g})\right]^{h /(h+2)} d \mathbf{g}\right]^{(h+2) / h} ; \text { then } \\
& K_{I}=\left[\int \frac{\exp \left\{-\frac{1}{2}\left(\mathbf{g}-\mu_{\mathbf{g}}\right)^{T} \frac{h}{h+2} C_{\mathbf{g}}^{-1}\left(\mathbf{g}-\mu_{\mathbf{g}}\right)\right\}}{\left[(\sqrt{2 \pi})^{h}\left|C_{\mathbf{g}}\right|^{1 / 2}\right]^{\frac{h}{h+2}}} d \mathbf{g}\right]^{\frac{h+2}{h}} \\
& =\left[\frac{(\sqrt{2 \pi})^{h}\left|\frac{h+2}{h} C_{\mathbf{g}}\right|^{1 / 2}}{(\sqrt{2 \pi})^{\frac{h^{2}}{h+2}}\left|C_{\mathbf{g}}\right|^{\frac{h}{2(h+2)}}} \int \mathbf{N}\left(\mu_{\mathbf{g}}, \frac{h+2}{h} C_{\mathbf{g}}\right) d \mathbf{g}\right]^{\frac{h+2}{h}} \\
& =(\sqrt{2 \pi})^{2}\left(\frac{h+2}{h}\right)^{\frac{h+2}{2}}\left|C_{\mathbf{g}}\right|^{\frac{1}{h}}
\end{aligned}
$$

Thus, using equality in (1), we get $\mathcal{D}_{\mathbf{g}}=$ $\left(2^{b_{g}}\right)^{-\frac{2}{h}} \frac{1}{\pi} \frac{h}{h+2}\left(\frac{h}{2} \Gamma\left(\frac{h}{2}\right)\right)^{\frac{2}{h}} K_{I}=\left(2^{b_{g}}\right)^{-\frac{2}{h}} K_{h}\left|C_{\mathbf{g}}\right|^{\frac{1}{h}}$.

\section{A. Mean Invariance to Quantization Distortion}

It is clear from (2) that the average quantization distortion is invariant to the mean vector of multivariate Gaussian pdf. It is a fact that the average distortion is a function of the pdf's spread and thus a function of the determinant of the covariance matrix for multivariate Gaussian distribution. In practice [13], 
the input vector can be easily mean removed (i.e., translated to zero mean vector) and then quantized using a VQ codebook that is optimized for zero mean vector pdf with the same covariance; at the decoder, the mean is restored by addition after decoding for reproduction. We note the importance of this mean invariant property to analyze the CSVQ method in the next section.

\section{Conditional PDF Based SPlit VQ}

For a multivariate Gaussian source, we derive the rate-distortion performance expression for conditional pdf-based split VQ (CSVQ) method using high rate theory. We assume that the source is quantized using $b$ bits/vector.

Let $\mathbf{X}$ be the $p$-dimensional vector that is multivariate Gaussian distributed as: $f_{\mathbf{X}}(\mathbf{x})=\mathbf{N}\left(\mu_{\mathbf{X}}, C_{\mathbf{X}}\right)$. We consider that the vector is split into $S$ number of subvectors, such that $\mathbf{X}=\left[\begin{array}{ll}\mathbf{X}_{1}^{T} \mathbf{X}_{2}^{T} \ldots \mathbf{X}_{S}^{T}\end{array}\right]^{T}$. Thus, the mean vector $\left(\mu_{\mathbf{X}}\right)$ and the covariance matrix $\left(C_{\mathbf{X}}\right)$ of the jointly Gaussian distribution, $f_{\mathbf{X}}(\mathbf{x})$, can be expressed in terms of the subvectors' means and covariances, as follows:

$$
\begin{aligned}
\mu_{\mathbf{X}} & =\left[\begin{array}{c}
\mu_{\mathbf{X}_{1}} \\
\mu_{\mathbf{X}_{2}} \\
\vdots \\
\mu_{\mathbf{X}_{S}}
\end{array}\right] \text {, and } \\
C_{\mathbf{X}} & =\left[\begin{array}{cccc}
C_{\mathbf{X}_{1} \mathbf{X}_{1}} & C_{\mathbf{X}_{1} \mathbf{X}_{2}} & \ldots & C_{\mathbf{X}_{1} \mathbf{X}_{S}} \\
C_{\mathbf{X}_{2} \mathbf{X}_{1}} & C_{\mathbf{X}_{2} \mathbf{X}_{2}} & \ldots & C_{\mathbf{X}_{2} \mathbf{X}_{S}} \\
\vdots & \vdots & \ddots & \vdots \\
C_{\mathbf{X}_{S} \mathbf{X}_{1}} & C_{\mathbf{X}_{S} \mathbf{X}_{2}} & \ldots & C_{\mathbf{X}_{S} \mathbf{X}_{S}}
\end{array}\right] .
\end{aligned}
$$

Let $p_{i}$ be the dimension of the $i$ th subvector $\mathbf{X}_{i}$, such that $\sum_{i=1}^{S} p_{i}=p$. Thus, the subcovariance matrix, $C \mathbf{X}_{i} \mathbf{X}_{j}$, is of dimension $p_{i} \times p_{j}$, along with the mean subvector, $\mu_{\mathbf{X}_{i}}$, of dimension $p_{i} \times 1$.

To exploit the conditional pdf, we sequentially quantize the subvectors of $\mathbf{X},\left\{\mathbf{X}_{i}\right\}_{i=1}^{S}$ [13]; i.e., $\mathbf{X}_{i}$ is quantized using the information about $\left\{\mathbf{X}_{1}, \mathbf{X}_{2}, \ldots, \mathbf{X}_{i-1}\right\}$. To quantize the $i$ th subvector, $\mathbf{X}_{i}$, let us define a vector concatenating the previous subvectors as

$$
\mathbf{Y}_{i}=\left[\mathbf{X}_{1}^{T} \mathbf{X}_{2}^{T} \ldots \mathbf{X}_{i-1}^{T}\right]^{T}
$$

In CSVQ, $\mathbf{X}_{i}$ is quantized using the past available information of $\mathbf{Y}_{i}$. The conditional pdf of $\mathbf{X}_{i}$ given the past information, i.e., $\mathbf{Y}_{i}=\mathbf{y}_{i}=\left[\mathbf{x}_{1}^{T} \mathbf{x}_{2}^{T} \ldots \mathbf{x}_{i-1}^{T}\right]^{T}$, is denoted as: $f_{\mathbf{X}_{i} \mid \mathbf{Y}_{i}}\left(\mathbf{x}_{i} \mid \mathbf{y}_{i}\right)$. Since $\mathbf{X}$ is jointly Gaussian, the conditional distribution of the $i$ th subvector, $\mathbf{X}_{i}$, given $\mathbf{Y}_{i}=\mathbf{y}_{i}$, is also a multivariate Gaussian distribution as follows [3]:

$$
f_{\mathbf{X}_{i} \mid \mathbf{Y}_{i}}\left(\mathbf{x}_{i} \mid \mathbf{y}_{i}\right)=\mathbf{N}\left(\mu_{\mathbf{X}_{i} \mid \mathbf{y}_{i}}, C_{\mathbf{X}_{i} \mathbf{X}_{i} \mid \mathbf{Y}_{i}}\right)
$$

where the parameters, the conditional mean vector, and the conditional covariance matrix are given as (see [3, Theorem 2.5.1])

$$
\begin{aligned}
\mu_{\mathbf{X}_{i} \mid \mathbf{y}_{i}} & =\mu_{\mathbf{X}_{i}}+C_{\mathbf{X}_{i} \mathbf{Y}_{i}} C_{\mathbf{Y}_{i} \mathbf{Y}_{i}}^{-1}\left(\mathbf{y}_{i}-\mu \mathbf{Y}_{i}\right) \\
C_{\mathbf{X}_{i} \mathbf{X}_{i} \mid \mathbf{Y}_{i}} & =C_{\mathbf{X}_{i} \mathbf{X}_{i}}-C_{\mathbf{X}_{i} \mathbf{Y}_{i}} C_{\mathbf{Y}_{i} \mathbf{Y}_{i}}^{-1} C_{\mathbf{Y}_{i} \mathbf{X}_{i}}
\end{aligned}
$$

The associated statistical parameters are given as follows [3]:

$$
\begin{aligned}
& \mu_{\mathbf{Y}_{i}}=\left[\begin{array}{c}
\mu_{\mathbf{X}_{1}} \\
\mu_{\mathbf{X}_{2}} \\
\vdots \\
\mu_{\mathbf{X}_{i-1}}
\end{array}\right], \quad C_{\mathbf{Y}_{i} \mathbf{X}_{i}}=\left[\begin{array}{c}
C_{\mathbf{X}_{1} \mathbf{X}_{i}} \\
C_{\mathbf{X}_{2} \mathbf{X}_{i}} \\
\vdots \\
C_{\mathbf{X}_{i-1} \mathbf{X}_{i}}
\end{array}\right] \\
& C_{\mathbf{X}_{i} \mathbf{Y}_{i}}=\left[\begin{array}{llll}
C_{\mathbf{X}_{i} \mathbf{X}_{1}} & \ldots & C_{\mathbf{X}_{i} \mathbf{X}_{i-1}}
\end{array}\right] \text { and } \\
& C_{\mathbf{Y}_{i} \mathbf{Y}_{i}}=\left[\begin{array}{ccc}
C_{\mathbf{X}_{1} \mathbf{X}_{1}} & \ldots & C_{\mathbf{X}_{1} \mathbf{X}_{i-1}} \\
C_{\mathbf{X}_{2} \mathbf{X}_{1}} & \ldots & C_{\mathbf{X}_{2} \mathbf{X}_{i-1}} \\
\vdots & \ddots & \vdots \\
C_{\mathbf{X}_{i-1} \mathbf{X}_{1}} & \ldots & C_{\mathbf{X}_{i-1} \mathbf{X}_{i-1}}
\end{array}\right] .
\end{aligned}
$$

We observe from (6) that the conditional mean is dependent on the value of $\mathbf{Y}_{i}=\mathbf{y}_{i}$, but the conditional covariance is a fixed parameter that is invariant to $\mathbf{y}_{i}$; hence, in (6), we use $\mathbf{y}_{i}$ for denoting the notation of conditional mean as $\mu_{\mathbf{X}_{i} \mid \mathbf{y}_{i}}$ but $\mathbf{Y}_{i}$ for the conditional covariance nota-

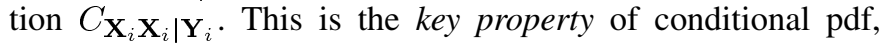
$f_{\mathbf{X}_{i} \mid \mathbf{Y}_{i}}\left(\mathbf{x}_{i} \mid \mathbf{y}_{i}\right)=\mathbf{N}\left(\mu_{\mathbf{X}_{i} \mid \mathbf{y}_{i}}, C_{\mathbf{X}_{i} \mathbf{X}_{i} \mid \mathbf{Y}_{i}}\right)$, which we use for analyzing the CSVQ method.

We already have noted that the average quantization distortion is mean invariant but only depends on the covariance. Thus, in the CSVQ method, the average distortion for the $i$ th subvector, $\mathbf{X}_{i}$, using the conditional distribution of (5), is given by [using (2)]

$$
\mathcal{D}_{C S V Q, i}\left(b_{i}\right)=\left(2^{b_{i}}\right)^{-\frac{2}{p_{i}}} K_{p_{i}}\left|C_{\mathbf{X}_{i} \mathbf{X}_{i} \mid \mathbf{Y}_{i}}\right|^{\frac{1}{p_{i}}}
$$

where $b_{i}$ is the bits allocated to the $i$ th subvector in CSVQ method, such that $\sum_{i=1}^{S} b_{i}=b$.

To minimize the average distortion in the CSVQ method, the total bits/vector (i.e., $b$ bits/vector) need to be allocated optimally among the subvectors. Using $b_{i}$ number of bits for the $i$ th subvector, the overall distortion for the full vector source can be written as

$$
\mathcal{D}_{\text {tot }}=\sum_{i=1}^{S} \mathcal{D}_{C S V Q, i}\left(b_{i}\right)
$$

The optimum bit allocation is decided by minimizing the overall distortion subject to the constraint of total bits as:

$$
\min _{b_{i}} \mathcal{D}_{t o t}=\sum_{i=1}^{S} \mathcal{D}_{C S V Q, i}\left(b_{i}\right), \text { subject to } \sum_{i=1}^{S} b_{i}=b \text {. }
$$

To allocate the bits optimally, we need to prove a lemma that is stated below.

Lemma 1: Determinant of a full rank covariance matrix is equal to the product of the determinants of respective conditional covariance matrices, given as

$$
\left|C_{\mathbf{X}}\right|=\prod_{i=1}^{S} \mid C_{\mathbf{X}_{i} \mathbf{X}_{i}\left|\mathbf{Y}_{i}\right|} .
$$

Proof: Using the definition of $C_{\mathbf{X}_{i} \mathbf{Y}_{i}}, C_{\mathbf{Y}_{i} \mathbf{Y}_{i}}, C_{\mathbf{Y}_{i} \mathbf{X}_{i}}$, and $C_{\mathbf{X}_{i} \mathbf{X}_{i} \mid \mathbf{Y}_{i}}$ [given in (7) and (6)], we can write

$$
\left|C_{\mathbf{Y}_{k+1} \mathbf{Y}_{k+1}}\right|=\left|\left[\begin{array}{ll}
C_{\mathbf{Y}_{k} \mathbf{Y}_{k}} & C_{\mathbf{Y}_{k} \mathbf{X}_{k}} \\
C_{\mathbf{X}_{k} \mathbf{Y}_{k}} & C_{\mathbf{X}_{k} \mathbf{X}_{k}}
\end{array}\right]\right| .
$$


Pre-multiplying $\left[C_{\mathbf{Y}_{k} \mathbf{Y}_{k}} C_{\mathbf{Y}_{k} \mathbf{X}_{k}}\right]$ with $C_{\mathbf{X}_{k} \mathbf{Y}_{k}} C_{\mathbf{Y}_{k} \mathbf{Y}_{k}}^{-1}$ and subtracting from $\left[C_{\mathbf{X}_{k} \mathbf{Y}_{k}} C_{\mathbf{X}_{k} \mathbf{X}_{k}}\right]$ (i.e., doing multiple row operations, which will not change the determinant), we get

$$
\begin{aligned}
\left|C_{\mathbf{Y}_{k+1} \mathbf{Y}_{k+1}}\right| & =\left|\left[\begin{array}{cc}
C_{\mathbf{Y}_{k} \mathbf{Y}_{k}} & C_{\mathbf{Y}_{k} \mathbf{X}_{k}} \\
\mathbf{0} & C_{\mathbf{X}_{k} \mathbf{X}_{k}}-C_{\mathbf{X}_{k} \mathbf{Y}_{k}} C_{\mathbf{Y}_{k}}^{-1} \mathbf{Y}_{k} C_{\mathbf{Y}_{k} \mathbf{X}_{k}}
\end{array}\right]\right| \\
& =\left|\left[\begin{array}{cc}
C_{\mathbf{Y}_{k} \mathbf{Y}_{k}} & C_{\mathbf{Y}_{k} \mathbf{X}_{k}} \\
\mathbf{0} & C_{\mathbf{X}_{k} \mathbf{X}_{k} \mid \mathbf{Y}_{k}}
\end{array}\right]\right| \\
& =\mid C_{\mathbf{Y}_{k} \mathbf{Y}_{k}|\cdot| C_{\mathbf{X}_{k} \mathbf{X}_{k} \mid \mathbf{Y}_{k}} \mid} .
\end{aligned}
$$

In the above derivation, we have used $C_{\mathbf{X}_{k}} \mathbf{X}_{k} \mid \mathbf{Y}_{k}=C_{\mathbf{X}_{k} \mathbf{X}_{k}}-$ $C_{\mathbf{X}_{k} \mathbf{Y}_{k}} C_{\mathbf{Y}_{k} \mathbf{Y}_{k}}^{-1} C_{\mathbf{Y}_{k} \mathbf{X}_{k}}$ [see (6)]. Now, by recursive relationship, we get

$$
\begin{aligned}
& \left|C_{\mathbf{X}}\right|=\left|C_{\mathbf{Y}_{S+1} \mathbf{Y}_{S+1}}\right| \\
& =\left|C_{\mathbf{Y}_{S} \mathbf{Y}_{S}}\right| \cdot\left|C_{\mathbf{X}_{S} \mathbf{X}_{S}\left|\mathbf{Y}_{S}\right|}\right|
\end{aligned}
$$

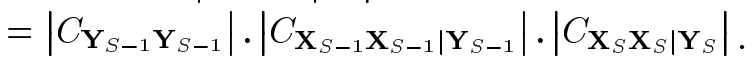

$$
\begin{aligned}
& =\prod_{i=1}^{S}\left|C_{\mathbf{X}_{i} \mathbf{X}_{i}\left|\mathbf{Y}_{i}\right|}\right|
\end{aligned}
$$

Now, we derive the performance expression of the CSVQ method using optimum bit allocation scheme as follows.

Theorem 1: The optimum inter subvector bit allocation for the CSVQ method that minimizes the overall distortion $\mathcal{D}_{\text {tot }}=$ $\sum_{i=1}^{S} \mathcal{D}_{C S V Q, i}\left(b_{i}\right)$, subject to the bit constraint $\sum_{i=1}^{S} b_{i}=b$, is given as [using the Lemma 1 of (11)]

$b_{i}=p_{i} \frac{b}{p}+\frac{p_{i}}{2} \log _{2}\left[\frac{\frac{1}{p_{i}} K_{p_{i}}\left|C_{\mathbf{X}_{i} \mathbf{X}_{i} \mid \mathbf{Y}_{i}}\right|^{\frac{1}{p_{i}}}}{\left[\prod_{j=1}^{S}\left(\frac{1}{p_{j}} K_{p_{j}}\right)^{p_{j}}\right]^{\frac{1}{p}}\left|C_{\mathbf{X}}\right|^{\frac{1}{p}}}\right], 1 \leq i \leq S$.

Proof:

$$
\begin{aligned}
& \mathcal{L}=\mathcal{D}_{\text {tot }}+\lambda\left(\sum_{i=1}^{S} b_{i}-b\right) \\
& =\sum_{i=1}^{S}\left(2^{b_{i}}\right)^{-\frac{2}{p_{i}}} K_{p_{i}}\left|C_{\mathbf{X}_{i} \mathbf{X}_{i} \mid \mathbf{Y}_{i}}\right|^{\frac{1}{p_{i}}}+\lambda\left(\sum_{i=1}^{S} b_{i}-b\right) .
\end{aligned}
$$

Using partial differentiation, $\frac{d \mathcal{L}}{d b_{i}}=0, \quad 1 \leq i \leq S$, we get

$$
b_{i}=-\frac{p_{i}}{2} \log _{2}\left[\frac{\lambda}{2 \ln 2}\right]-\frac{p_{i}}{2} \log _{2}\left[\frac{p_{i}}{K_{p_{i}} \mid C_{\mathbf{X}_{i} \mathbf{X}_{i}\left|\mathbf{Y}_{i}\right|^{\frac{1}{p_{i}}}}}\right] \text {. }
$$

Summing $\forall i$ and using $\sum_{i=1}^{S} p_{i}=p, \sum_{i=1}^{S} b_{i}=b$, we get

$$
-\frac{1}{2} \log _{2}\left[\frac{\lambda}{2 \ln 2}\right]=\frac{b}{p}+\frac{1}{p} \sum_{i=1}^{S} \frac{p_{i}}{2} \log _{2}\left[\frac{p_{i}}{K_{p_{i}} \mid C_{\mathbf{X}_{i} \mathbf{X}_{i}\left|\mathbf{Y}_{i}\right|^{\frac{1}{p_{i}}}}}\right] .
$$

Substituting $-(1 / 2) \log _{2}[\lambda / 2 \ln 2]$ appropriately and using

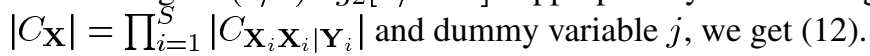

Theorem 2: Using the optimum inter subvector bit allocation of (12), the minimum overall distortion for the CSVQ method is given as

$$
\mathcal{D}_{C S V Q}=\left(2^{b}\right)^{-\frac{2}{p}}\left[p\left[\prod_{i=1}^{S}\left(\frac{1}{p_{i}} K_{p_{i}}\right)^{p_{i}}\right]^{\frac{1}{p}}\right]\left|C_{\mathbf{X}}\right|^{\frac{1}{p}} .
$$

Proof: Using the optimum bit allocation of Theorem 1 (12), the minimum overall distortion

$$
\begin{aligned}
\mathcal{D}_{C S V Q}= & \left.\mathcal{D}_{\text {tot }}\right|_{\text {min }}=\sum_{i=1}^{S} \mathcal{D}_{C S V Q, i}\left(b_{i}\right) \\
= & \sum_{i=1}^{S}\left(2^{b_{i}}\right)^{-\frac{2}{p_{i}}} K_{p_{i}} \mid C_{\mathbf{X}_{i} \mathbf{X}_{i}\left|\mathbf{Y}_{i}\right|^{\frac{1}{p_{i}}}} \\
= & \sum_{i=1}^{S} 2^{-\frac{2}{p_{i}}}\left[p_{i} \frac{b}{p}+\frac{p_{i}}{2} \log _{2}\left[\frac{\left.\frac{1}{p_{i}} K_{p_{i}}\left|C_{\mathbf{X}_{i}} \mathbf{x}_{i}\right| \mathbf{Y}_{i}\right|^{\frac{1}{p_{i}}}}{\left[\prod_{j=1}^{S}\left[\frac{1}{p_{j}} K_{p_{j}}\right]^{p_{j}}\right]^{\frac{1}{p}}\left|C_{\mathbf{X}}\right|^{\frac{1}{p}}}\right]\right] \\
& \times\left. K_{p_{i}}\left|C_{\mathbf{X}_{i} \mathbf{X}_{i}\left|\mathbf{Y}_{i}\right|^{\frac{1}{p_{i}}}}\left[2^{b}\right)^{-\frac{2}{p}}\left[p\left[\prod_{j=1}^{S}\left[\frac{1}{p_{j}} K_{p_{j}}\right]^{p_{j}}\right]^{\frac{1}{p}}\right]\right| C_{\mathbf{X}}\right|^{\frac{1}{p}} .
\end{aligned}
$$

\section{SPLIT VQ}

In this section, we derive the rate-distortion performance expression for the traditional split vector quantization (SVQ) method where the subvectors are quantized independently. We already have noted that the $i$ th subvector, $\mathbf{X}_{i}$, is multivariate Gaussian distributed as: $f_{\mathbf{X}_{i}}\left(\mathbf{x}_{i}\right)=\mathbf{N}\left(\mu_{\mathbf{X}_{i}}, C_{\mathbf{X}_{i} \mathbf{X}_{i}}\right)$. Thus, in the SVQ method, the average distortion for the $i$ th subvector, $\mathbf{X}_{i}$, is given as [using (2)]

$$
\mathcal{D}_{S V Q, i}\left(c_{i}\right)=\left(2^{c_{i}}\right)^{-\frac{2}{p_{i}}} K_{p_{i}}\left|C_{\mathbf{X}_{i} \mathbf{X}_{i}}\right|^{\frac{1}{p_{i}}}
$$

where $c_{i}$ is the bits allocated to the $i$ th subvector in the SVQ method, such that $\sum_{i=1}^{S} c_{i}=b ; b$ is the total allocated bits/ vector to quantize the vector source, like CSVQ.

To minimize the overall distortion in the SVQ method, the total available $b$ bits/vector need to be allocated optimally among the split subvectors. Using $c_{i}$ number of bits for $\mathbf{X}_{i}$, the overall distortion for the full vector source is written as

$$
\mathcal{D}_{\text {tot }}=\sum_{i=1}^{S} \mathcal{D}_{S V Q, i}\left(c_{i}\right) .
$$

The optimum bit allocation is decided by minimizing the overall distortion subject to the constraint of total bits as

$$
\min _{c_{i}} \mathcal{D}_{t o t}=\sum_{i=1}^{S} \mathcal{D}_{S V Q, i}\left(c_{i}\right), \text { subject to } \sum_{i=1}^{S} c_{i}=b .
$$


We derive the performance expression of SVQ method using optimum bit allocation scheme as follows.

Theorem 3: The optimum inter subvector bit allocation for the SVQ method that minimizes the overall distortion $\mathcal{D}_{\text {tot }}=$ $\sum_{i=1}^{S} \mathcal{D}_{S V Q, i}\left(c_{i}\right)$, subject to the bit constraint $\sum_{i=1}^{S} c_{i}=b$, is given as

$$
\begin{aligned}
& c_{i}=p_{i} \frac{b}{p}+\frac{p_{i}}{2} \log _{2}\left[\frac{\frac{1}{p_{i}} K_{p_{i}} \mid C_{\left.\mathbf{X}_{i} \mathbf{X}_{i}\right|^{\frac{1}{p_{i}}}}\left[\prod_{j=1}^{S}\left(\frac{1}{p_{j}} K_{p_{j}}\right)^{p_{j}}\right]^{\frac{1}{p}}\left[\prod_{j=1}^{S} \mid C_{\left.\mathbf{X}_{j} \mathbf{X}_{j} \mid\right]^{\frac{1}{p}}}\right.}{]}\right. \\
& 1 \leq i \leq S \text {. }
\end{aligned}
$$

Proof: Using the similar approach for proving Theorem 1.

Theorem 4: Using the optimum inter subvector bit allocation of (17), the minimum overall distortion for SVQ method is given as

$$
\begin{aligned}
\mathcal{D}_{S V Q} & =\left.\mathcal{D}_{\text {tot }}\right|_{\text {min }}=\sum_{i=1}^{S} \mathcal{D}_{S V Q, i}\left(c_{i}\right) \\
& =\left(2^{b}\right)^{-\frac{2}{p}}\left[p\left[\prod_{i=1}^{S}\left(\frac{1}{p_{i}} K_{p_{i}}\right)^{p_{i}}\right]^{\frac{1}{p}}\right]\left[\prod_{i=1}^{S}\left|C_{\mathbf{X}_{i} \mathbf{X}_{i}}\right|\right]^{\frac{1}{p}} .
\end{aligned}
$$

Proof: Using the similar approach for proving Theorem 2.

\section{CODING Gain OF CSVQ OVER SVQ}

In this section, we show the coding gain of the CSVQ method over the SVQ method. To show the coding gain, we need to prove another lemma, which is stated below.

Lemma 2: Determinant of a full-rank covariance matrix is less than or equal to the product of the determinants of respective subcovariance matrices, given as

$$
\left|C_{\mathbf{X}}\right| \leq \prod_{i=1}^{S}\left|C_{\mathbf{X}_{i} \mathbf{X}_{i}}\right|
$$

Proof: Let $A$ be a real, positive definite matrix given as

$$
A=\left[\begin{array}{ll}
A_{11} & A_{12} \\
A_{21} & A_{22}
\end{array}\right]
$$

where $A_{i j}, 1 \leq i, j \leq 2$ are the block submatrices. Then, following [1], it is known that $|A| \leq\left|A_{11}\right| \cdot\left|A_{22}\right|$.

Using the same notations for proving Lemma 1 in (11), we get

$$
\begin{aligned}
\left|C_{\mathbf{Y}_{k+1} \mathbf{Y}_{k+1}}\right| & =\left|\left[\begin{array}{ll}
C_{\mathbf{Y}_{k} \mathbf{Y}_{k}} & C_{\mathbf{Y}_{k} \mathbf{X}_{k}} \\
C_{\mathbf{X}_{k} \mathbf{Y}_{k}} & C_{\mathbf{X}_{k} \mathbf{X}_{k}}
\end{array}\right]\right| \\
& \leq\left|C_{\mathbf{Y}_{k} \mathbf{Y}_{k}}\right| \cdot\left|C_{\mathbf{X}_{k} \mathbf{X}_{k}}\right| .
\end{aligned}
$$

Thus, by recursive relationship, we get

$$
\begin{aligned}
\left|C_{\mathbf{X}}\right| & =\left|C_{\mathbf{Y}_{S+1} \mathbf{Y}_{S+1}}\right| \\
& \leq\left|C_{\mathbf{Y}_{S} \mathbf{Y}_{S}}\right| \cdot\left|C_{\mathbf{X}_{S} \mathbf{X}_{S}}\right| \\
& \leq\left|C_{\mathbf{Y}_{S-1}} \mathbf{Y}_{S-1}\right| \cdot\left|C_{\mathbf{X}_{S-1} \mathbf{X}_{S-1}}\right| \cdot\left|C_{\mathbf{X}_{S} \mathbf{X}_{S}}\right| \\
& \leq \prod_{i=1}^{S}\left|C_{\mathbf{X}_{i} \mathbf{X}_{i}}\right|
\end{aligned}
$$

Now, we show the coding gain of the CSVQ method over the SVQ method as follows.

Coding Gain: Using Theorem 2 [(13)], Theorem 4 [(18)]. and Lemma 2 [(19)], the coding gain of CSVQ over SVQ is given as

$$
\mathcal{G}_{C S V Q \_S V Q}=\frac{\mathcal{D}_{S V Q}}{\mathcal{D}_{C S V Q}}=\frac{\left[\prod_{i=1}^{S}\left|C_{\mathbf{X}_{i} \mathbf{X}_{i}}\right|\right]^{\frac{1}{p}}}{\left|C_{\mathbf{X}}\right|^{\frac{1}{p}}} \geq 1 .
$$

Thus, the CSVQ method performs better than the SVQ method. If there are no correlations between the subvectors, then all the

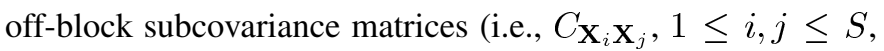
$i \neq j$ ) are zeros in the full covariance matrix, $C_{\mathbf{X}}$; in this case, only the equality holds in the above (20), and the CSVQ method does not provide any coding gain over the SVQ method.

\section{CONCLUSION}

Using high rate quantization theory, the R/D performance analysis is carried out for the recently proposed CSVQ and traditional SVQ methods. For a multivariate Gaussian source, the analysis allows us to show the coding gain of the CSVQ method over the SVQ method in the high-rate regime.

\section{REFERENCES}

[1] E. F. Beckenbach and R. Bellman, "Inequalities," Theorem 7, ch. 2, Second revised printing, Springer-Verlag. New York, 1965.

[2] Y. Yamada, S. Tazaki, and R. M. Gray, "Asymptotic performance of block quantizers with difference distortion measures," IEEE Trans. Inf. Theory, vol. IT-26, no. 1, pp. 6-14, Jan. 1980.

[3] T. W. Anderson, An Introduction to Multivariate Statistical Analysis, 2nd ed. New York: Wiley, 1984, ch. 2.

[4] R. M. Gray, Source Coding Theory. Dordrecht, The Netherlands: Kluwer, 1990.

[5] A. Gersho and R. M. Gray, Vector Quantization and Signal Compression. Norwell, MA: Kluwer, 1992.

[6] K. K. Paliwal and B. S. Atal, "Efficient vector quantization of LPC parameters at 24 bits/vector," IEEE Trans. Acoust., Speech, Signal Process., vol. 1, no. 1, pp. 3-14, Jan. 1993.

[7] R. Lefebvre, R. Salami, C. Laflamme, and J. P. Adoul, "High quality coding of wideband audio signals using transform coded excitation (TCX)," in Proc. ICASSP, 1994, pp. 193-196.

[8] J. H. Chen and D. Wang, "Transform predictive coding of wideband speech signals," in Proc. ICASSP, 1996, pp. 275-278.

[9] P. Hedelin and J. Skoglund, "Vector quantization based on Gaussian mixture models," IEEE Trans. Speech Audio Process., vol. 8, no. 4, pp. 385-401, Jul. 2000.

[10] M. Y. Kim and W. B. Kleijn, "KLT-based adaptive classified VQ of the speech signal," IEEE Trans. Speech Audio Process., vol. 12, no. 3, pp. 277-289, May 2004.

[11] F. Norden and T. Eriksson, "On split quantization of LSF parameters," in Proc. ICASSP, May 2004, vol. 1, pp. I-157-I-160.

[12] S. Chatterjee and T. V. Sreenivas, "Sequential split vector quantization of LSF parameters using conditional pdf," in ICASSP-2007, accepted for publication.

[13] S. Chatterjee and T. V. Sreenivas, "Conditional PDF-based split vector quantization of wideband LSF parameters," IEEE Signal Process. Lett., accepted for publication. 